\title{
New thinking about thinking, part two. Theoretical articles for Alzheimer's \& Dementia
}

Khachaturian AS, Hayden KM, Mielke MM, Tang Y, Lutz MW, Gold M, Kukull WA, Mohs R, Gauthier S, Molinuevo JL, Zetterberg H, Khachaturian ZS.

There is one question often asked during scientific meetings, business travel, or dinner parties that is just as easily and equally posed by members of the research community, the general public or by friends and family: what's new in Alzheimer's research? Today there is growing interest from readers into the insights derived from new therapy development, systems biology and network modeling analytics. There is also increasing public health awareness of the neeed for translation and potential benefits of the application of precision medicine, community/population level data, and big omics data into effective interventions, meaningful health policy, and robust technologies to better monitor, assess and control disease progression. These varied expressions of Alzheimer's translational research perhaps represent the best examples of what's new.

The amplification of translational research and the resulting knowledge now present an important publication challenge. Alzheimer's \& Dementia receives many manuscripts that represent efforts from multi-disciplinary teams who push science forward, beyond traditional research domains. These reports often describe new technologies, different methodologies, or recalibrated analytical approaches. These papers comprise an emerging and growingcollection of the published literature and in a unique and paradoxical sense, a new area of concern for the field.

In 2005, Carl R. Woese ${ }^{1}$ highlighted this potentially looming problem in scholarly writing, "science is impelled by two main factors, technological advance and a guiding vision (overview). Without a guiding vision, there is no road ahead; the science becomes an engineering discipline, concerned with temporal practical problems." He continued, "a society that permits biology to become an engineering discipline, that allows the science to slip into the role of changing the living world without trying to understand it, is a danger to itself."

The Journal is enthusiastic about the exciting recent calls for a more pluralistic neuroscience. ${ }^{2}$ This view encompasses a balanced consideration of both the theoretical and the experimental aspects of brain-behavior research, particularly in the aging individual. While there is no question about the importance of reporting novel data or new investigative techniques, there is now a growing imperative for authors to articulate their new insights, ideas, or hypotheses within the context of a summation of previous investigations and conceptual frameworks.

To support this aim, Alzheimer's \& Dementia is describing the specifications, format and layout for articles focused on a theoretical perspective. The new format for theoretical articles will provide authors the option to submit manuscripts such as those that synthesize early or pilot data into new testable conceptual models. Manuscripts might also describe theoretical frameworks or hypotheses spanning any of the following 
generic areas including (but not limited to) biology, chemistry, clinical/medical interventions, behavior/neuropsychology, social sciences, nursing, health economics, health services research and public policy. This editorial presents a new standard format for the theoretical article type and provides some guidelines for preparation of these types of manuscripts for publication in Alzheimer's \& Dementia.

Theoretical articles, and specifically the abstract, should be written for a diverse audience so that the central research question, the expression of the hypotheses, the important research challenges, and the linkages with existing ideas, conceptual frameworks, or theories are easily understood. Manuscripts should follow the format below and include each element and sub-element listed:

1. Structured abstract

2. Objective

3. Background

a. Historical evolution

b. Rationale

4. New or Updated hypothesis

a. Early experimental or observational data

b. Future experiments and validation studies

5. Major challenges for the hypothesis

6. Linkage to other major theories

Each of these elements and sub-elements will be described in further detail below. In addition, the other required items include key words, references, acknowledgements, conflicts, and funding sources. Length may not exceed 3,500 words (excluding the abstract, references, technical appendices, figures, and tables), a maximum of 50 references, no more than six figures, boxes, tables or some combination of six figures, boxes and tables.

The following section provides an outline as a general guide for organizing the six elements for a theoretical article. To help illustrate, the example used below discusses the important elements for a future hypothesis on neuroinflammation. Please note this general format comes with a strong caveat: individual topics may require some modification to this template.

1. STRUCTURED ABSTRACT FOR THE THEORETICAL ARTICLE: The abstract should contain the elements presented above for the theoretical article format including, the following headings, Objective, Background, New/Updated Hypothesis, Major Challenges for the Hypothesis, and Linkage to Other Major Theories. Each heading should have short sentences that summarizes the individual elements as described below. The structured abstract should not exceed 350 words.

Main Body of the Paper: 
2. OBJECTIVE: In a brief paragraph orient the reader regarding the main purpose of the paper: what is the basic (e.g., take-home) message or headline news for the proposed hypothesis.

For example: "This paper is a proposal for an update of the Hypothesis on Inflammatory Mechanism in Neurodegeneration-Dementia-Alzheimer syndrome (abbreviated hereafter as the Hypothesis) based on emerging novel evidence. The present draft intends to: a) promote new thinking about the biological substrates and the origins of neurodegeneration and, b) solicit input from other key leaders in the field to amend further and finalize the present version of the Hypothesis. This effort aims to reassess the role of inflammation in neurodegeneration and to identify potential disease-modifying interventions and/or risk-reducing therapeutic strategies that target the mechanistic relationships between inflammation and neuronal and/or glial cell functions/functioning."

3. BACKGROUND: This element contains a brief narrative about the background, evolution, and data providing the rationale for the reevaluation of the hypothesis, proposed rejection or, revision of the hypothesis. In presenting a brief history, there should be some discussion of how the historically postulated mechanisms may play a central role in neurodegeneration. Depending on the depth of the literature, a more extensive review of this discourse may be attached as an appendix. After the background and historical review, clearly state the theoretical rationale. Please note, in some instances the construction of a robust and logical theoretical argument may require the use of several declarative sentences that then conclude with a single interrogative sentence.

The following example might be paraphrased to fit the proposed hypothesis: "The customary paradigms of therapy development, essentially derived from current ideas and existing models of etiology, have not yielded any effective treatments during the last three decades. In addition, the recent string of unsuccessful clinical trials has provided further credence to the growing recognition that there are major gaps in understanding the biology of Alzheimer's disease. This breakdown in treatment strategies, based on current ideas, indicates the need to examine the problem from a different perspective. It is very likely that the present notions on the pathogenesis of dementia Alzheimer's disease, which provide the mechanistic rationale for typical models of drug discoverydevelopment, may be insufficient. Such a drastic transformation in thinking will need new conceptual models that integrate a wide range of biochemical mechanisms that underlie the pathogenesis of various forms/types of neurodegeneration, dementia, and Alzheimer's disease."

4. NEW OR UPDATED HYPOTHESIS: This element should provide a concise articulation of the major claims the new or reformulated hypothesis. This discussion should enumerate the key postulates and may include not only speculations about putative mechanisms but also strategies to confirm [i.e., crucial experiments] the predictions of the revised hypothesis. Revisions to existing hypotheses may require a higher burden of proof to supplant an established idea. 
In addition to this discussion, manuscripts will need to include two sub-elements 1) Early Experimental or Observational Data and 2) Future Experiments and Validation Studies. For the sub-element, Early Experimental or Observational Data, manuscripts should review and describe early-, pilot-, simulated-, or meta-data derived from experimental or observational research. This section should follow the familiar, albeit condensed, format of a typical research paper's methods and results sections. If such data do not exist, the authors will need to explain why and provide some other basis for justification.

For the sub-element, Future Experiments and Validation Studies, manuscripts must explain the specific predictions that the updated hypothesis will offer along with the detailed outlines of potential experiments necessary either to confirm these predictions (or falsifies the hypothesis).

\section{MAJOR CHALLENGES FOR THE HYPOTHESIS:}

The aim for this section is a careful review of barriers that must be surmounted, or the technical challenges necessary to adequately test the hypothesis. Given the strong possibility that the tools, instruments and other methods necessary to conduct future pivotal experiments do not exist, the submitted manuscripts should describe major challenges. Discuss whether, and if so, how a new/revised hypothesis will account for or address critical questions and common challenges confronting all major theories of Alzheimer's dementia.

These questions include:

Question \#1 Association with Age: Does the putative theory explain the relationship between the biology of aging and the biology of Alzheimer pathology?

Question \#2 Risk factors: Will the putative theory explain the relationships between known risk or protective factors and the neurobiological mechanisms (e.g. genetic mutations, susceptibility genes, epigenetic modifications, increase or decrease in cognitive reserve, structural modification of the brain, etc.) by which they modify risk?

Question \#3 Progression: Does the putative theory account for the patterns of progression of the disease (e.g., from limbic to neocortical), the asymmetry of progression, and the relationship (probably non-linear) to changing levels of clinical severity? Can the putative theory describe the mechanism of disease progression through contiguity, axonal transport, prion-like mechanisms etc.?

Question \#4 Sequence of events: Does the putative theory predict the observable sequence of measurable or observable events that contribute to disease? Does the putative theory explain how and when the clinical manifestations, relate to the pathophysiology? 
Question \#5 Selective vulnerability: Does the putative theory provide a plausible explanation for the topological specificity of early lesions associated with the syndrome?

Question \#6 Pathogenic interactions: Does the putative theory account for the interactions between elements believed to be key to pathology?

Question \#7 Mixed pathology: Does the putative theory account for mixed pathologies and comorbid conditions (e.g., Lewy bodies, vascular pathology, TDP-43)?

Question \#8 Multiple clinical phenotypes: Does the putative theory provide an explanation for the clinical heterogeneity of the syndrome, especially the non-amnestic manifestations of Alzheimer pathology?

Question \#9 Biomarkers: Does the putative theory account for the existing puzzles in diagnostic biomarker relationships? It is also of interest to know whether the theory offers new candidate biomarkers that can also track risk and prognosis.

Question \#10 Translational potential: Can the putative theory lead to novel therapeutic targets or diagnostic technologies?

In those instances where the new or reformulated hypothesis is not able to provide adequate answers, these could be listed as future challenges for the hypothesis.

6. LINKAGE TO OTHER MAJOR THEORIES: A key question for any new/revised hypothesis is whether it provides an account for linkages or mechanistic relationships with other major ideas on dementia. Referencing our original example on inflammation, this section may include some discussion and/or speculation on the functional relationship of inflammation with the universe of other theories or the temporal location of inflammation related events in the sequence pathogenic processes-paths suggested by different theories e.g., amyloid, tau, calcium, vascular changes, and metabolic dysfunction? A future challenge for a reformulated inflammation hypothesis might be the postulation of specific mechanistic relationships to account for synaptic loss and/or possible explanations for similar or different (if any) mechanistic paths to synaptic loss resulting from or mediated via inflammation vs. amyloid or tau-induced pathobiology ${ }^{3}$.

This new theoretical article type requires authors to conduct a significant and thorough examination of theory. To further assist in this process, we offer two key concepts that should be considered for the reframing of a hypothesis: These recommendations, discussed below, should provide a common framework to either develop or reassess ideas and thinking on the origins of neurodegeneration. The rationale for this approach is self-explanatory in the narrative below.

Recommendation \#1: Development of any new or revised hypothesis must address the common requirement of accounting for mechanisms that influence the performance of a neuron and/or the functions of a network or system. Although various ideas on dementia 
and Alzheimer may start with very different assumptions, any viable theory must account for the deficits in functional connectivity of various networks associated with neurodegeneration.

Persistent waning in synaptic transmission, continuing pruning of dendritic arbors, massive loss of synapses, and ineffective repair and homeostatic functions, are believed to be proximal cellular abnormalities in the expression of clinical features of virtually all neurodegenerative disorders, including dementia - Alzheimer's disease. Thus, a vital focus for any hypothesis should be an explanation of how the performance of a neuron and neuronal circuits as systems is affected.

The rationale for this emphasis is based on the core premise that progressive decline in the performance of normal functions of neurons is the most proximal event common to all chronic brain disorders associated with neurodegeneration. The reformulation of any hypothesis should consider stressing this notion as a key component for explaining the complex interactions among the array of molecular mechanisms for maintaining optimal functionality of individual neurons and neuronal networks as systems. The hypothesis should explain how early age-related upstream alterations at the molecular and cellular levels [mediated by inflammatory mechanisms in our example] might affect the performance of neurons and their ability to cope with environmental stressors.

Recommendation \#2: Development of any new or revised hypothesis requires that the theory should adopt the concept of a final common path as a means for describing key triggering events that might initiate the cascade of events leading to a common endpoint.

This concept is based on the premise that there are several alternative mechanisms for deregulating or disrupting the activities of a neuron. There are differing paths toward the decline in a neuron's functionality - performance. For example, there is emerging evidence that inflammatory mechanisms play a central role or somehow are involved in the disruption of multiple pathways, which set the stage for neuro-degenerative processes leading to the deterioration of performance in a neural network. This idea is intended to foster new conceptual models that integrate a wide range of biochemical mechanisms that underlie the pathogenesis of various forms/types of neurodegeneration, dementia, and Alzheimer's disease.

In summary, we wish to encourage new thinking about Alzheimer's disease. The theoretical article is a framework for communicating new, revised, or emerging concepts. The presented format, including new headings, should guide the author in the organization and structure of the theoretical article. The questions listed under item 5, Major Challenges for the Hypothesis, are given to help authors think through potential conceptual problems that may be encountered in the development of a new idea. The two final recommendations are essentially caveats that authors should consider in proposing or revising a hypothesis. This guide should aid efforts to communicate new thinking about thinking. We hope these concepts are thought-provoking and look forward to your ideas. 
Draft Editorial: New Thinking on Thinking Part 2 // MAY 18, 2018

Khachaturian AS et al.

DO NOT COPY OR DISTRIBUTE WITHOUT PERMISSION

\section{Ara S. Khachaturian, Ph.D. (Corresponding Author)}

Executive Editor

Alzheimer's \& Dementia: The Journal of the Alzheimer's Association

451 Hungerford Drive, \#119-355

Rockville, MD 20850

W: 301-309-6730

F: (844) 309-6730

E: adj_xed@kra.net

http://www.alzheimersanddementia.org

http://adj.edmgr.com

Kathleen M. Hayden, Ph.D.

Associate Professor

Wake Forest School of Medicine

Department of Social Sciences and Health Policy

Division of Public Health Sciences

Sticht Center for Healthy Aging and Alzheimer's Prevention

Winston-Salem, NC

khayden@wakehealth.edu

Michelle M. Mielke, Ph.D.

Professor of Epidemiology | Department of Health Sciences Research

Professor of Neurology

Mayo Clinic College of Medicine

Rochester, MN

mielke.michelle@mayo.edu

Yi Tang, M.D., Ph.D.

Associate Professor

Department of Neurology

Xuan Wu Hospital

Beijing, China

tangyixw@163.com

Michael W. Lutz, Ph.D.

Assistant Professor of Neurology

Department of Neurology

Duke University School of Medicine

Durham, NC

michael.lutz@duke.edu

Michael Gold, M.D.

Vice-President, Development Neurosciences 
Draft Editorial: New Thinking on Thinking Part 2 // MAY 18, 2018

Khachaturian AS et al.

DO NOT COPY OR DISTRIBUTE WITHOUT PERMISSION

AbbVie, Inc.

North Chicago, IL

michael.gold@abbvie.com

Walter A. Kukull, Ph.D.

Professor, Epidemiology

School of Public Health

University of Washington

Seattle, WA

kukull@uw.edu

Richard Mohs, Ph.D.

Senior Associate Editor

Alzheimer's \& Dementia: The Journal of the Alzheimer's Association

Chicago, IL

mohsrichard@yahoo.com

Serge Gauthier, M.D.

Director

McGill Centre for Studies in Aging

Montreal, QC

serge.gauthier@mcgill.ca

José Luis Molinuevo M.D., Ph.D.

Scientific Director

Alzheimer Prevention Program

BarcelonaBeta Brain Research Center

Fundació Pasqual Maragall

Barcelona, Spain

jlmolinuevo@barcelonabeta.org

Zaven S. Khachaturian, Ph.D.

Editor-in-Chief

Alzheimer's \& Dementia: The Journal of the Alzheimer's Association

Rockville, MD

adj@kra.net

1 Woese, C.R. (2004). A new biology for a new century. Microbiol. Mol. Biol.

Rev.

68, 173-186.

2 Krakauer, J.W., Ghazanfar, A.A., Gomez-Marin, A., MacIver, M.A., and

Poeppel, D. (2017). Neuroscience Needs Behavior: Correcting a Reductionist Bias. Neuron 93(3): $480-490$. 
Draft Editorial: New Thinking on Thinking Part 2 // MAY 18, 2018

Khachaturian AS et al.

DO NOT COPY OR DISTRIBUTE WITHOUT PERMISSION

3 Hong S, Beja-Glasser VF, Nfonoyim BM, Frouin A, Li S, Ramakrishnan S, Merry KM, Shi Q, Rosenthal A, Barres BA, Lemere CA, Selkoe DJ, Stevens

B. Complement and microglia mediate early synapse loss in Alzheimer mouse models. Science. 2016 Mar 31 